\title{
2-D Locally Regularized Tissue Strain Estimation From Radio-Frequency Ultrasound Images: Theoretical Developments and Results on Experimental Data
}

\author{
Elisabeth Brusseau*, Jan Kybic, Jean-François Déprez, and Olivier Basset
}

\begin{abstract}
In this paper, a 2-D locally regularized strain estimation method for imaging deformation of soft biological tissues from radio-frequency ( $R F$ ) ultrasound (US) data is introduced. Contrary to most 2-D techniques that model the compression-induced local displacement as a 2-D shift, our algorithm also considers a local scaling factor in the axial direction. This direction-dependent model of tissue motion and deformation is induced by the highly anisotropic resolution of RF US images. Optimal parameters are computed through the constrained maximization of a similarity criterion defined as the normalized correlation coefficient. Its value at the solution is then used as an indicator of estimation reliability, the probability of correct estimation increasing with the correlation value. In case of correlation loss, the estimation integrates an additional constraint, imposing local continuity within displacement and strain fields. Using local scaling factors and regularization increase the method's robustness with regard to decorrelation noise, resulting in a wider range of precise measurements. Results on simulated US data from a mechanically homogeneous medium subjected to successive uniaxial loadings demonstrate that our method is theoretically able to accurately estimate strains up to $17 \%$. Experimental strain images of phantom and cut specimens of bovine liver clearly show the harder inclusions.
\end{abstract}

Index Terms-Elastography, optimization, strain estimation, ultrasound (US).

\section{INTRODUCTION}

$\mathbf{U}$ TLRASOUND elastography is an emerging imaging modality dedicated to the investigation of the elastic properties of soft biological tissues [1]. This technique is of fundamental interest for the clinical diagnosis of various diseases, since the development of a pathological process is often correlated with local changes in tissue stiffness [2], [3]. Elastography might therefore provide useful information for

Manuscript received February 3, 2007; revised February 28, 2007. This work was supported by a grant from the Scientific Committee of the INSA-Lyon, France. The work of J. Kybic was supported by the Grant Agency of the Czech Academy of Sciences under Grant 1ET101050403. Asterisk indicates corresponding author.

*E. Brusseau is with CREATIS INSA-Lyon; Université de Lyon; Université Lyon 1; CNRS UMR 5220; INSERM U630; F-69621 Villeurbanne, France.

J.-F. Déprez and O. Basset are with CREATIS INSA-Lyon; Université de Lyon; Université Lyon 1; CNRS UMR 5220; INSERM U630; F-69621 Villeurbanne, France.

J. Kybic is with the Czech Technical University, Prague 2 12135, Czech Republic.

Digital Object Identifier 10.1109/TMI.2007.897408 early detection and characterization of various pathologies and for patient treatment follow up [4]-[12]. In practical terms, the tissue is deformed by applying a static load or by generating low-frequency mechanical waves that propagate within the medium. Tissue internal displacements and deformations are then locally estimated from acquired radio-frequency (RF) ultrasound (US) images, by partitioning the US data into many overlapping regions of interest (ROI) and by evaluating, within each ROI, the positional variations induced by the stress. Deformation analysis may be completed by the reconstruction of mechanical parameters such as the Young's modulus.

This paper focuses on estimating the strain field in static elastography, where compression is applied with the probe [Fig. 1(a)]. In elastography, strains must be estimated with high accuracy since clinicians' diagnosis as well as the quality of mechanical parameter reconstruction are directly related to these estimations. Until recently, the most commonly used strain estimation techniques were 1-D. They analyze only the 1-D variations generated by the stress application, and occurring along the US beam propagation axis (axial axis). Essentially, two approaches have been reported in the literature. The first one groups together techniques that compute the axial strain as the spatial gradient of the displacements that the tissue locally experiences when compressing forces are applied [13]-[17]. The local displacement is assumed to be a simple axial translation, resulting in a shift of the corresponding 1-D ROI within RF signals. It is estimated by a correlation analysis. These methods are accurate for small deformations. However, they rapidly fail with increasing strains, because they ignore the signal shape variation induced by the physical compression of the medium and responsible for decorrelation [18], [19].

This observation has led to the development of techniques that also take into account a signal shape modification. Specifically, these methods consider that when applying the load the tissue locally undergoes a 1-D deformation comparable to a compression [20]-[22]. The signal after compression is locally assumed to be a shifted and spatially-scaled replica of the original signal. By estimating the local axial scaling factor between the precompression and postcompression signals, the deformation profile is deduced. Using scaling factors provides estimation methods that are much more robust in terms of decorrelation noise and increases the range of accuracy in strain measurements.

The major limitation of all previously mentioned methods is their 1-D character. When a biological medium is subjected to 


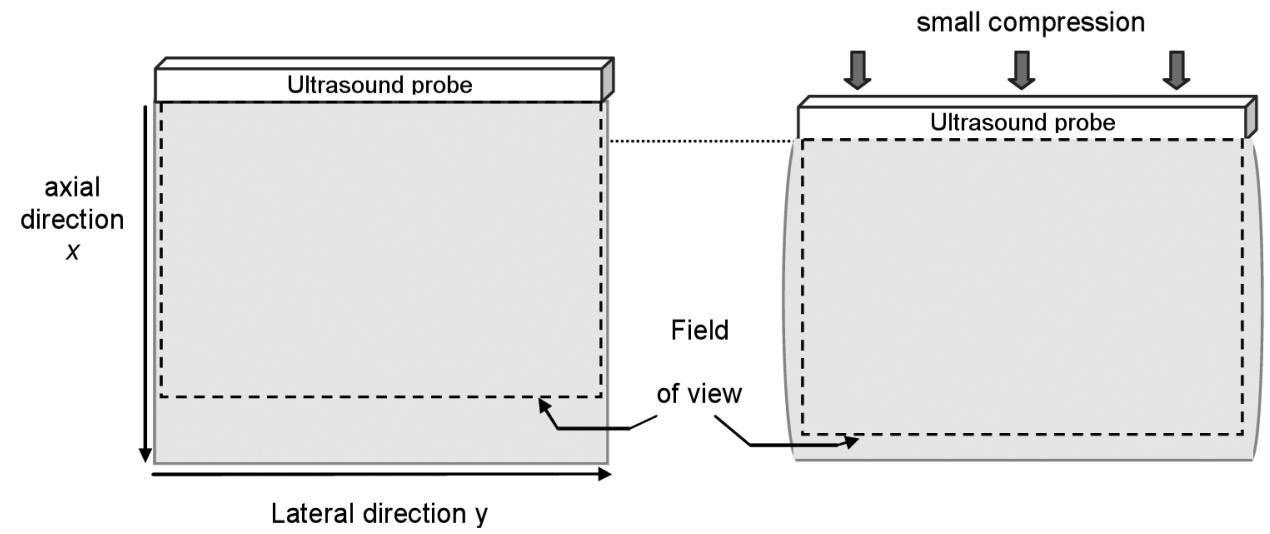

(a)
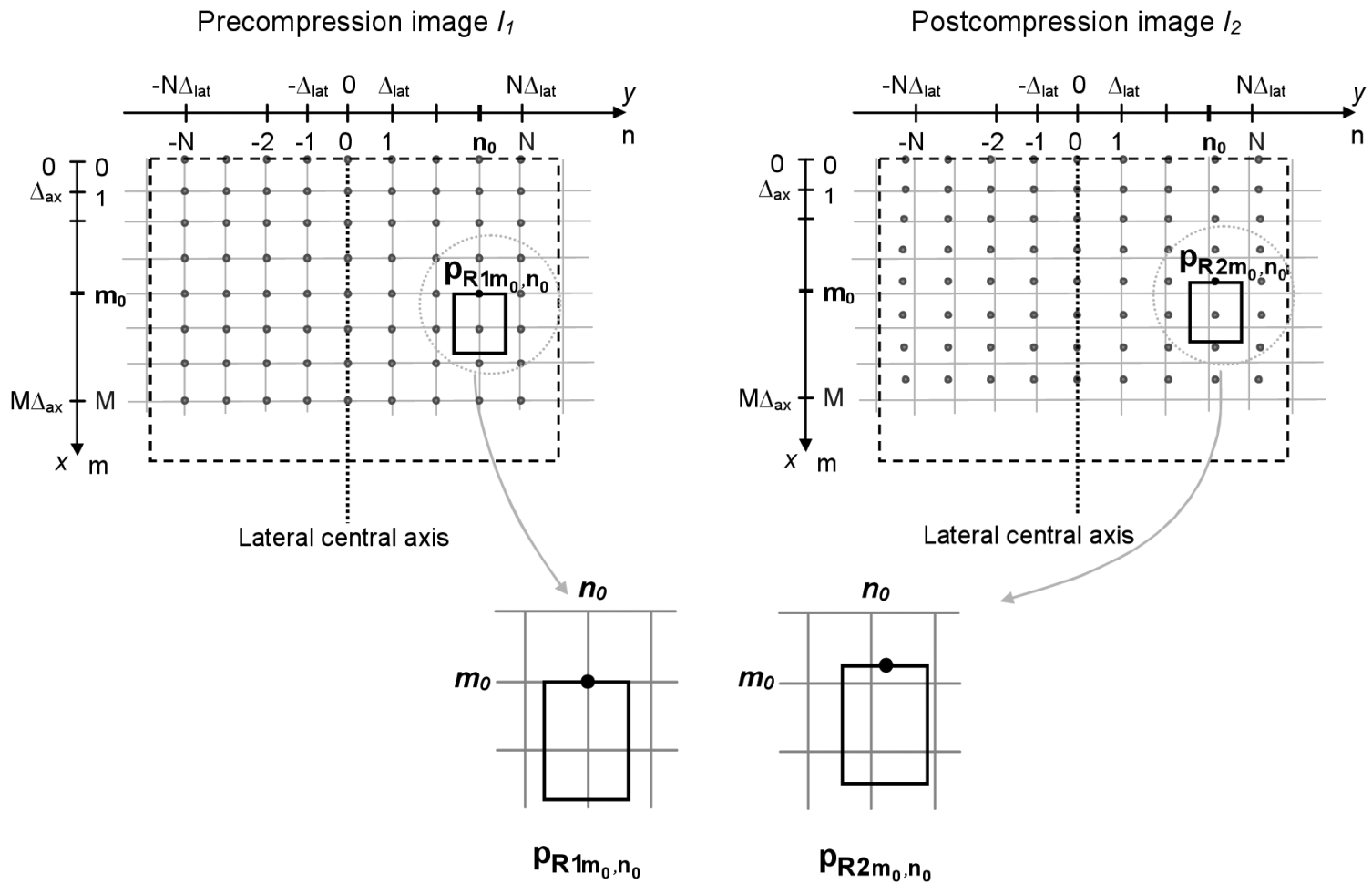

(b)

Fig. 1. (a) Geometry of the acquisition; left: tissue in its initial configuration, right: tissue under load. Dashed line delimits the field of view of the US probe. (b) Illustration of the ROI adaptive displacement. While the successive positions of $R_{1}$ in the precompression image $I_{1}$ covers a regular grid, in the postcompression image $I_{2}, R_{2}$ is adaptively displaced according to the effects on its position produced by the deformation of surrounding regions.

an axial compression, it also naturally undergoes a lateral and azimuthal expansion. Ideally, estimating the strain should take into account the 3-D tissue motion. However, because clinical US scanners typically provide only 2-D images, in this paper we consider the problem of 2-D strain estimation from 2-D RF US data acquisitions.

Only a few 2-D techniques have been reported to date. Most of them model 2-D local displacement as a translation in both axial and lateral (perpendicular to the US beam's propagation axis in the image plane) directions, and then compute strain estimates as the displacement gradient. The simplest approach is the 2-D speckle tracking [23]. Unfortunately, it lacks robustness and accuracy because of compression-induced signal decorrelation and the coarse lateral spacing between adjacent signals. An alternate approach improving the estimation of lateral displacements was proposed by Chen et al. [24]. Lateral tracking can conceptually perform better if lateral phase information, similar to that in the axial direction, is present in the final 2-D correlation function. The synthetic lateral phase is generated numerically by splitting the analytic signal spectrum with respect to zero frequency in the lateral direction into up and down halves. The 2-D displacement is then determined as phase zero-crossings in both dimensions. Unfortunately, the improvement in lateral tracking is limited only to regions where strain is less than $1 \%$, and becomes insignificant with increasing strain magnitude [24]. The main reason is the signal decorrelation induced by tissue motion.

To help restore the coherence of echoes prior to cross correlation, several techniques have been developed. Konofagou et 
al. [25] describe a global axial stretch of the RF signals and a weighted interpolation method, operating between these signals, to track the lateral displacement. Companding methods consisting of joint operations of compression and expansion of echo fields have also been introduced [26], [27]. The 2-D companding technique integrates a two-scale analysis, both global and local. Global companding is initially performed to compensate for the average deformations and displacements, assuming the medium is incompressible and spatially uniform in elasticity. In a second-pass process, local companding is achieved by estimating, for many overlapping kernels, the 2-D motion between precompression and postcompression echo fields, and by shifting echo waveforms accordingly. Finally, cross correlation is applied along the direction of the beam propagation to measure the residual axial displacement. The latter is then added to the displacements induced by the global and local compandings, before computing the gradient to form the strain image. Such algorithms provide more accurate axial strain estimates. Nevertheless, the scaling factors applied to compensate for signal decorrelation are not optimal, because they are not specifically adapted to each ROI considered.

Maurice et al. [28], [29] suggest estimating the 2-D strain field by determining the linear transformation that locally exists between the initial and deformed images. In practical terms, strain parameters, corresponding to local scaling factors, are estimated as the arguments that minimize the mean square error between a precompression ROI and its deformed version compensated for the assumed deformation parameters. The authors underline that, prior to the strain parameter estimation, the 2-D translation that inherently occurs between the precompression and postcompression 2-D ROIs needs to be removed. This compensation is performed using correlation techniques and may therefore lack accuracy over highly strained regions and corrupt the strain estimation.

A recent study [30] pointed out that data alone may be insufficient to solve the ambiguities caused by the loss of echo coherence, and therefore integrating a priori knowledge into the motion estimation process might be needed. The 2-D displacement field is estimated by locally minimizing an energy equation, imposing constraints of echo amplitude conservation and displacement field smoothness. Such an algorithm prevents noisy strain fields. However, it only considers constant shifts (not scaling) and uses a spatially constant regularization parameter that may excessively smooth the boundaries.

The aim of this paper is to present a 2-D strain estimation method with improved performance. This method estimates the axial strain while considering lateral displacement. The algorithm is based on an iterative and adaptive process, appropriate to investigating a medium subjected to a wide range of strains. Achieving maximum accuracy requires processing that adequately fits the local strain variations. However, as described by [30] echo coherence may be lost and the proposed technique needs to be able to overcome this problem. Therefore, the method estimates the parameters, in terms of 2-D shifts and time-scaling factor, by matching precompressed and postcompressed 2-D acoustical footprints as closely as possible. This optimal parameter estimation is performed through the constrained maximization of a similarity criterion. Unlike described techniques, the value of the similarity criterion at the solution is used as an indicator of estimation reliability, and over regions where variations in the signal have led to ambiguities or incoherence, deformation parameters are recomputed by locally imposing smoothness constraints.

This paper is organized as follows: the theoretical framework and the technique implementation are described in Section II, followed by results on simulated and experimental data in Section III. Section IV provides a discussion of the results along with concluding remarks.

\section{METHOD}

\section{A. Deformation Model}

An increase in the range of accurate estimates was recently demonstrated [20]-[22] for 1-D techniques, when not only shifts but also scaling factors of acoustical footprints are considered in the strain estimation. In a first approximation, the signal after deformation in the direction of the US wave propagation can thus be considered as a locally shifted and scaled replica of the signal prior to deformation.

Axially compressed, biological media also undergo an expansion along the lateral direction. Therefore, similar effects, local shifts and scaling factors, might be considered in the lateral direction as well. However, US RF data are characterized by a highly anisotropic resolution. The resolution in the axial direction is very fine, primarily determined by the US carrier frequency, whereas the lateral resolution is much rougher, limited by the acoustic aperture size. For these reasons, the scaling factor will be considered only in the axial direction. The assumed relation between the precompression and postcompression images, $I_{1}$ resp. $I_{2}$, can be locally expressed as follows:

$$
I_{1}(x, y)=I_{2}(\alpha x+\tau, y+d)
$$

where $(x, y)$ are the axial and lateral variables, respectively, $\alpha$ is the axial scaling (compression) factor, and $\tau$, and $d$ the axial and lateral displacements, respectively. $\alpha, \tau$ and $d$ are spatially slowly varying parameters.

\section{B. Method Description}

As previously mentioned, locally estimating strains implies considering a small ROI in $I_{1}$ that is moved throughout the entire image, and for each of its positions, its deformed version in $I_{2}$ is determined and the compression-induced variations are analyzed.

Let us consider any region of interest $R_{1}$ at the position $p_{R 1}$ in $I_{1}$. The physical compression of the medium has two impacts on this ROI.

- The first one is the variation in its position, resulting from the deformation of the surrounding tissues. Let us denote $R_{2}$ the deformed version of $R_{1}$ in $I_{2}$. The compression of regions located between $R_{1}$ and the probe produces an axial shift between $R_{1}$ and $R_{2}$, described by the parameter $\tau$ in (1). Similarly, surrounding medium deformation will induce a lateral shift between $R_{1}$ and $R_{2}$. This lateral shift, denoted $v$, represents the major contribution of $d$ (1).

- The second one is its specific deformation, a function of its own mechanical parameters. 
These shifts $\tau$ and $v$ can be compensated for if an adequate strategy to track the ROI $R_{2}$ corresponding to $R_{1}$ is adopted. In this case, only two parameters remain to be estimated for each ROI, those relative to its specific deformation, namely the scaling factor $\alpha$ and a small-magnitude residual lateral shift $u$ (such that $d=u+v$ ).

The algorithm implemented includes the following four subtasks, described in greater detail hereafter:

1) two-dimensional adaptive displacement of ROIs;

2) joint estimation of the axial scaling factor $\alpha$ and the lateral shift $u$;

3) field representation;

4) local regularization.

1) Regions of Interest 2-D Adaptive Displacement: To compensate for $\tau$ and $v$, and, therefore, consider the same physical tissue region before and after deformation, $R_{1}$ and $R_{2}$ are displaced simultaneously and adaptively in both images. In the precompression image $I_{1}, R_{1}$ describes a succession of vertical (axial) sweeps from the probe downwards, and from the image center [identified by the central axis, see Fig. 1(b)] toward lateral extremities. Along the axial direction, $R_{1}$ is displaced with a constant step $\Delta_{\mathrm{ax}}$. Laterally, the sweeps' interdistance is also constant, and denoted $\Delta_{\text {lat }}$. Significant overlap is maintained in both directions. The set of $R_{1}$ positions therefore covers a regular grid.

While regularly moving $R_{1}$ in $I_{1}, R_{2}$ is displaced adaptively in $I_{2}$ by considering the effects of the deformation of surrounding tissues on its position. Its axial position is calculated by accumulation of the axial compression of regions located between the probe and its current position. Its lateral position differs from that of $R_{1}$ by a global shift, which is the sum of the lateral shifts that the adjacent regions undergo, regions located between the current position and the central axis.

More formally, let $p_{R 1}$ be the position of $R_{1}$ in $I_{1}$ and $p_{R 2}$ the position of $R_{2}$ in $I_{2}$ after compensation for $\tau$ and $v$. These positions are defined with respect to the middle point of the ROI top boundary [see Fig. 1(b)]. Let us identify by a horizontal (lateral) index $n$ and a vertical (axial) index $m$ the set of positions of $R_{1}$ in $I_{1}$, denoted $p_{R 1 m, n}$ and of $R_{2}$ in $I_{2}\left(p_{R 2 m, n}\right)$. The corresponding axial and lateral shifts are denoted $\tau_{m, n}$ and $v_{m, n}$, respectively. The positions $p_{R 1 m, n}$ and $p_{R 2 m, n}$ have the following coordinates, show in (2) and (3) at the bottom of the page, where $\operatorname{sgn}(n)$ represents the sign function. $\alpha_{k, n}$ is the estimated axial scaling factor for the region located at $p_{R 1 k, n}$ and $u_{m, k}$, the estimated lateral signed distance between $R_{1}$ and $R_{2}$ located at $p_{R 1 m, k}$ and $p_{R 2 m, k}$, respectively.

Specific conditions must be mentioned. At the probe-medium interface, the axial shift is inherently equal to zero. This is indicated by the condition $\alpha_{-1, n}=0$. Moreover, along the first vertical sweep, prior to the first lateral displacement estimation, $R_{1}$ and $R_{2}$ are initialized, centered on the lateral central axis, and thus have the same lateral position. Finally, parameters $\tau_{m, n}$ and $v_{m, n}$ correspond locally to the signed difference of positions $p_{R 2 m, n}$ and $p_{R 1 m, n}$ in the axial and lateral directions, respectively

$$
\tau_{m, n}=\left[\sum_{k=0}^{m} \alpha_{k-1, n}-m\right] \cdot \Delta_{\mathrm{ax}}
$$

and

$$
v_{m, n}=\sum_{k=0}^{n} u_{m, k-\operatorname{sgn}(n)} .
$$

Finally let $L$ be the axial length and $W$ the lateral width of the ROIs. $R_{1}$ at $p_{R 1 m, n}\left(m \Delta_{\mathrm{ax}}, n \Delta_{\text {lat }}\right)$ is the part of $I_{1}(x, y)$ such that

$$
\begin{array}{r}
R_{1}=I_{1}\left(x, y \mid m \cdot \Delta_{\mathrm{ax}} \leq x \leq m \cdot \Delta_{\mathrm{ax}}+L-1, n \cdot \Delta_{\text {lat }}\right. \\
\left.-\left\lfloor\frac{W}{2}\right\rfloor \leq y \leq n \cdot \Delta_{\text {lat }}-\left\lfloor\frac{W}{2}\right\rfloor+W-1\right)
\end{array}
$$

where $\lfloor\cdot\rfloor$ represents the floor function. The width $W$ is in practical terms a number of signal segments. The spatial variables for ROIs will still be denoted $x$ and $y$ such that $R_{1}(x, y)=R_{1}$. A similar relation characterizes the postcompression region $R_{2}$.

Once the adaptive ROI displacement has been performed, only the axial scaling factor $\alpha_{m, n}$ and the small magnitude lateral displacement $u_{m, n}$ remain to be estimated.

2) Axial Scaling Factor and Lateral Shift Joint Estimation: $R_{1}$ at $p_{R 1 m, n}$ and $R_{2}$ at $p_{R 2 m, n}$ having been determined, the optimal parameters $(\alpha, u)$ are estimated by optimizing an objective function based on a similarity criterion. They are determined as the arguments that maximize the normalized correlation coefficient between the initial region $R_{1}$ and its deformed version $R_{2}$, when the latter is compensated for according to these parameters.

Unlike classical approaches, we take advantage of the fact that, in elastography, biological tissue compression is small,

$$
\begin{aligned}
P_{R 1 m, n}\left(m \Delta_{\mathrm{ax}}, n \Delta_{\mathrm{lat}}\right) & m=0,1, \ldots, M \text { and } n=-N, \ldots,-1,0,1, \ldots N \\
P_{R 2 m, n}\left(\sum_{k=0}^{m} \alpha_{k-1, n} \cdot \Delta_{\mathrm{ax}}, n \Delta_{\mathrm{lat}}+\sum_{k=0}^{n} u_{m, k-\operatorname{sgn}(n)}\right) \text { with } \alpha_{-1, n} & =0 \\
\text { and } u_{m,-1} & =0 \text { for } n \geq 0 \\
u_{m, 1} & =0 \text { for } n<0 \\
\operatorname{sgn}(n) & =1 \text { for } n \geq 0,-1 \text { otherwise }
\end{aligned}
$$


resulting in an expected small range of admissible parameter values. Consequently, we use constrained optimization, which increases the robustness of the estimation.

Maximizing an objective function is equivalent to minimizing the opposite of this function. In terms of minimization, the constrained nonlinear programming problem to be solved is

$$
\begin{array}{r}
{[\hat{\alpha}, \hat{u}]=\arg \min _{\alpha, u} f(\alpha, u)} \\
\text { subject to: } \alpha_{\min } \leq \alpha \leq \alpha_{\max } \\
u_{\min } \leq u \leq u_{\max }
\end{array}
$$

where $f$ (see the equation at the bottom of the page) is the opposite of the normalized correlation coefficient between $R_{1}$ and $R_{2}, \bar{R}_{1}$ and $\bar{R}_{2}$ the mean values of $R_{1}(x, y)$ and $R_{2}(x, y)$, respectively, and $x$ and $y$ the axial and lateral variables.

Defining $t=(\alpha, u)$, the minimization problem can be rewritten as

$$
\begin{gathered}
{[\hat{t}]=\arg \min _{t} f(t)} \\
\text { subject to: At } \leq b
\end{gathered}
$$

where the matrix $A$ and the vector $b$ contain the coefficients associated with parameters and bounds, respectively. The necessary conditions for a feasible point $t^{*}$ to be a local minimum of (7) are

$$
\begin{array}{ll}
G 1: & A t^{*} \leq b \text { with } \hat{A} t^{*}=\hat{b} \\
G 2: & Z^{T} \nabla f\left(t^{*}\right)=0 \Leftrightarrow \nabla f\left(t^{*}\right)+A^{T} \lambda^{*}=0 \\
G 3: & \lambda_{i}^{*} \geq 0, \forall i \\
G 4: & \lambda_{i}^{*}\left(A_{i} t^{*}-b_{i}\right)=0, \forall i \\
G 5: & Z^{T} H\left(t^{*}\right) Z \text { is positive semi-definite }
\end{array}
$$

where $\hat{A}$ is the submatrix of $A$ containing the coefficients of the constraints active at $t^{*}$ (those on bounds) and $\hat{b}$ the subvector of $b$, such that $\hat{A} t^{*}=\hat{b} . Z$ is the matrix whose columns form a basis for the set of vectors orthogonal to the rows of $\hat{A}$. $Z^{T} \nabla f\left(t^{*}\right)$ and $Z^{T} H\left(t^{*}\right) Z$ are the projected gradient and Hessian at $t^{*}$, respectively. The Lagrange multipliers are denoted by $\lambda_{i}$. $G 2, G 3$, and $G 4$ define the Kuhn-Tucker conditions.

To solve (7), we use an optimization algorithm based on the sequential quadratic programming (SQP) methodology [31]. Such methods can be viewed as the natural extension of Newton (or quasi-Newton) techniques to constrained optimization setting. This iterative procedure consists in modeling the defined problem at a given approximate solution $t_{k}$, by a quadratic programming (QP) subproblem, and then in using the subproblem solution to construct a better approximation $t_{k+1}$ as follows:

$$
t_{k+1}=t_{k}+\rho_{k} d_{k}
$$

where $\rho_{k}$ is the step length and $d_{k}$ the QP solution that defines the descent direction. The QP subproblem solved at each iteration takes the following form:

$$
\begin{aligned}
& \min _{d_{t}} \nabla f\left(t_{k}\right)^{T} \cdot d_{t}+\frac{1}{2} d_{t}^{T} \cdot H\left(t_{k}\right) d_{t} \\
& \text { subject to: } A\left(d_{t}+t_{k}\right) \leq b
\end{aligned}
$$

where $d_{t}=t-t_{k}, \nabla f$ is the objective function gradient computed by a finite-difference approximation and $H$ is the Hessian of $f$. The latter is initialized to the Identity matrix and a positive-definite approximation is iteratively built through BFGS updates [32].

This quadratic subproblem is solved for $d_{t}$, using an active set strategy. It is an iterative procedure that aims at identifying which inequality constraints will become active at the solution [(8,G1)], determining the subspace $Z$ of feasible search directions. More precisely, having a prediction of this subspace $Z_{j}$, a typical QP iteration $j$ consists in computing the descent direction following the iterative scheme:

$$
t_{j+1}=t_{j}+\zeta_{j} \cdot d_{j}
$$

where the initial value of $t_{j}$, namely $t_{0}$, corresponds to $t_{k}$, and where $d_{j}=Z_{j} \cdot p$ is determined by solving the equation

$$
\begin{aligned}
\left(Z_{j}^{T} H\left(t_{0}\right) Z_{j}\right) p & =-Z_{j}^{T} \nabla f_{j} \\
\text { with } \nabla f_{j} & =\nabla f\left(t_{0}\right)+H\left(t_{0}\right) \cdot\left(t_{j}-t_{0}\right)
\end{aligned}
$$

$\zeta_{j}$ is a step length for which only two choices are possible. A step of unity along $d_{j}$ is the exact step to the minimum. If it can be taken without constraint violation, Lagrange multipliers are computed and if all positive $[(8, G 3)]$, the QP minimum is achieved. Otherwise, if one multiplier is negative, the associated constraint is deleted from the active set, $Z_{j}$ is updated, and the process iterated. Finally, if there is an inequality constraint blocking the way toward the minimum, $\zeta_{j}$ is less than unity and fixed to the distance to the nearest constraint. The blocking constraint is added to the active set, involving the update of $Z_{j}$, and a new iteration is performed.

Once the descent direction $d_{k}$ is determined, the step length $\rho_{k}$ is varied until a sufficient decrease in the objective function is obtained. It is upper bounded by the distance to the nearest constraint in the direction $d_{k}$.

SQP methods, like Newton's method, are only guaranteed to find a local solution of (6). The small size of the feasible region considered, discussed later in the Results section, drastically reduces the occurrence of local minima but does not eliminate it. To make the algorithm converge toward the solution sought, optimization can be initialized with configurations that are close to the global minimum [33]. Since displacement and strain fields are continuous, the optimal parameter vector for one region can

$$
f(\alpha, u)=\frac{-\sum\left(R_{1}(x, y)-\bar{R}_{1}(x, y)\right) \cdot\left(R_{2}\left(\frac{x}{\alpha}, y+u\right)-\bar{R}_{2}\left(\frac{x}{\alpha}, y+u\right)\right)}{\sqrt{\sum\left(R_{1}(x, y)-\bar{R}_{1}(x, y)\right)^{2}} \sqrt{\sum\left(R_{2}\left(\frac{x}{\alpha}, y+u\right)-\bar{R}_{2}\left(\frac{x}{\alpha}, y+u\right)\right)^{2}}}
$$


be used as the initial value for the minimization process in a neighboring region.

More formally, let us consider $R_{1}$ at $p_{R 1 m, n}$ and $R_{2}$ at $p_{R 2 m, n}$. The ROI adaptive displacement results in a lateral shift $u$ of small magnitude. It will, therefore, be initialized to 0 . On the other hand, the scaling factor will be initialized to the optimal scaling factor obtained for the region immediately above the current position, such that

$$
\begin{aligned}
\alpha_{0, n}^{(0)} & =\gamma \text { mean value of the feasible axial range } \\
\alpha_{m, n}^{(0)} & =\hat{\alpha}_{m-1, n} m>0
\end{aligned}
$$

where the superscript (0) indicates initialization of the iterative process.

Even if suitable, these initializations are not sufficient to prevent the algorithm from being trapped in a local minimum. In particular, for regions at the probe-medium interface, the scaling factor is arbitrarily initialized to the mean value of the feasible axial range. To avoid keeping parameter vectors that would have been wrongly estimated, a correction procedure is introduced. It uses the similarity criterion value to assess whether the estimate is potentially incorrect. Estimates are suspected of being erroneous when their normalized correlation coefficient remains below a threshold $R_{\text {threshold }}$, since the probability of correct estimation is higher if this coefficient is closer to 1 . Once an insufficiently reliable estimate has been detected, a better parameter vector value is sought by initializing $N b$ new minimization processes from $\mathrm{Nb}$ points uniformly spread within the parameter domain. The parameter vector retained is the one leading to the highest correlation coefficient.

3) Field Representation: To sum up, by describing a succession of vertical sweeps, $R_{1}$ covers the entire image $I_{1}$ and for each of its positions $p_{R 1 m, n}$, its corresponding ROI $R_{2}$ in $I_{2}$ is determined and the parameter vector $\hat{t}_{m, n}=\left[\hat{\alpha}_{m, n} ; \hat{u}_{m, n}\right]$ estimated. The axial compression factor field $\hat{\boldsymbol{\alpha}}=\left\{\hat{\alpha}_{m, n},(m, n)=[0, \ldots, M] \times[-N, \ldots, N]\right\}$ and the lateral displacement field $\hat{\mathbf{u}}=$ $\left\{\hat{u}_{m, n},(m, n)=[0, \ldots, M] \times[-N, \ldots, N]\right\} \quad$ are thus obtained.

The field that is specifically interesting is the axial strain field $\varepsilon$. Since axial strain corresponds to the relative change in length of an infinitesimal line element of the medium along the axial direction, $\hat{\varepsilon}$ can be easily computed from the axial compression factor field $\hat{\alpha}$ [34]. It is determined as $\hat{\varepsilon}=\left\{\hat{\varepsilon}_{m, n},(m, n)=[0, \ldots, M] \times[-N, \ldots, N]\right\}$, with

$$
\hat{\varepsilon}_{m, n}=\hat{\alpha}_{m, n}-1 \text {. }
$$

Note that with this formula, the strain field follows the established convention that the strain is negative for a compression and positive for a dilatation. However, since in elastography we always work in compression and for simplification purposes, we will display the opposite of the axial strain field.

Since most strain imaging techniques estimate the axial shift distribution, we have decided to also represent the axial displacement field. This field corresponds to the integration of the axial strain field along the axial direction. In practical terms, this operation is performed by a cumulative summation along that direction. The axial displacement distribution $\hat{\tau}$ is thus defined as

$$
\begin{aligned}
\hat{\tau}=\left\{\hat{\tau}_{m, n}=\sum_{i=0}^{m} \hat{\varepsilon}_{i, n} \cdot \Delta_{\mathrm{ax}}\right. & \\
& (m, n)=[0, \ldots, M] \times[-N, \ldots, N]\} .
\end{aligned}
$$

With the convention used in (14), it clearly appears that the axial displacement is negative when a compression is observed. Therefore, similarly to the axial strain field, we will display the opposite of the axial displacement field, knowing that these displacements result from the compression of the medium.

Finally, the correlation coefficient map is presented as well, providing information on the reliability of the results. The closer to 1 the correlation is, the higher the probability of correct estimation.

4) Local Regularization: The three steps, Sections II-B1, II-B2, and II-B3, result in initial displacement and strain fields with a local indicator of the estimation reliability. Generally, these fields exhibit small areas of unreliable estimates because of locally large or out-of-plane motion or insufficiently strong signals. A low correlation coefficient does not signify an erroneous estimation, but it means that the estimate cannot be trusted and further investigation with additional information is necessary.

Let us denote by $\Omega$ the set of parameter vectors whose estimation has led to a high normalized correlation coefficient $\hat{R}_{m, n}$, higher than a threshold value $R_{\text {reg }}$. These vectors have a strong probability of being correctly estimated and are retained unchanged

$$
\begin{aligned}
\Omega=\left\{\hat{t}_{m, n}=\left[\hat{\alpha}_{m, n}, \hat{u}_{m, n}\right]\right. & \\
(m, n) & =[0, \ldots M] \times[-N, \ldots, 0, \ldots N] \\
\mid \hat{R}_{m, n} & \left.=-f\left(\hat{t}_{m, n}\right) \geq R_{\mathrm{reg}}\right\} .
\end{aligned}
$$

On the other hand, parameter vectors that do not belong to $\Omega$ are considered unreliable and need to be recomputed by introducing a priori information. Since displacement and strain are continuous 2-D fields, the new parameter vector estimation imposes a local smoothness constraint with surrounding parameter values belonging to $\Omega$.

To correct a parameter vector $\hat{t}_{m, n}$, we first consider a neighborhood $V$ of this estimate in the parameter field. $V$ is initialized to the 8 neighboring vectors with respect to the discrete grid $m, n$. Those belonging to $\Omega$ are selected and if they represent at least a quarter of the neighboring vectors, the neighborhood is retained. If not, the neighborhood $V$ grows uniformly until at least $25 \%$ of the parameters belong to $\Omega$. Only the parameter vectors at the intersection of $V$ and $\Omega$ are retained and their weighted average value, $t_{\text {average, }}$ is computed. Weights are the correlation coefficients associated with the parameter vectors. $\hat{t}_{m, n}$ is then estimated anew using the following minimization 
[shown in (17) at the bottom of the page] where $\mu$ is a positive regularization factor. This factor as well as the parameter $R_{\text {reg }}$ have been adjusted empirically.

Considering neighborhoods containing at least $25 \%$ reliable values avoids correcting an estimate with the information of only one isolate point, and ensures continuity with close, wellestimated regions.

The proposed regularization process is dedicated to the correction of localized ambiguities or estimation errors. It is, therefore, expected that initial displacement and strain fields obtained from Sections II-B1, II-B2, and II-B3 are of enough good quality, in other words that areas of insufficiently reliable estimation are small. However, the advantage of our technique is its ability to discriminate which areas of the displacement and strain fields need to be recomputed and to select only reliable information to redo the estimation.

\section{RESULTS}

\section{A. Results on Simulated Data}

The technique performance was first assessed on simulated data. This required modeling the tissue motion under compression and the US image formation.

1) Displacement-Field and Image-Formation Models: Simulated media are assumed to exhibit homogeneous echogenicity, making any lesion undetectable with standard US imaging. Acoustically, they are modeled as a set of scatterers that are spatially uniformly distributed and whose acoustical amplitudes are normally distributed within the range $[-1,1]$. Deforming the medium implies scatterer interdistance variations, depending on the mechanical properties of the region they belong to. Depending on the complexity of the medium's mechanical properties, the new location for the scatterers is computed with closed-form equations or using a finite element modeling software.

The image formation in this study is assumed to be a linear space-invariant operation. US RF images are generated by convolving the acoustical scatterer distributions with the point spread function (PSF) of the imaging system [28]. The PSF shape is direction-dependent (Fig. 2). Axially, it is modeled as a 7-MHz cosine function, modulated by a Gaussian. In the lateral direction, the PSF is considered to be Gaussian-shaped. The PSF shape is completed by introducing a slight curvature,

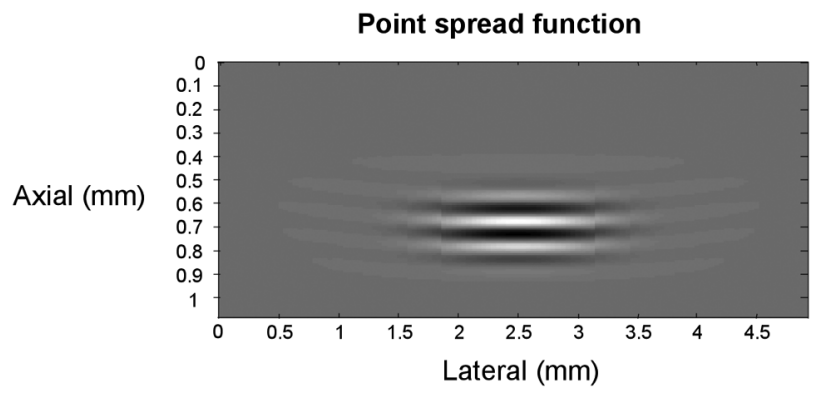

Fig. 2. Representation of the simulated point spread function.

making it similar to those measured experimentally [35]. Finally, the sampling frequency has been set to $100 \mathrm{MHz}$. The generated images are composed of $128 \mathrm{RF}$ lines.

2) Homogeneous Mechanical Media Results: To assess the accuracy of the presented method and to determine some of its parameters, the deformation of a $40 \times 40 \times 40-\mathrm{mm}^{3}$ cube of mechanically homogeneous material under successive uniaxial loadings with negligible friction was investigated. In particular, the section for which no out-of-plane motion occurs was considered. The medium was assumed to be linear elastic, isotropic, and incompressible (Poisson's ratio, $\nu=0.5$ ). Nineteen compression levels inducing $0.5 \%$, and from $1 \%$ to $18 \%$ (by $1 \%$ steps) of averaged axial strains $(\varepsilon)$ were considered. As previously mentioned, simulated medium deformation involves moving the acoustical scatterers accordingly. In this simple case, scatterer motion in the plane considered is described by the following equations:

$$
\left\{\begin{array}{l}
x_{p_{\text {nnew }}}=(1+\varepsilon) x_{p} \\
y_{p \text { nnew }}=(1-\nu \varepsilon) y_{p}
\end{array}\right.
$$

where $\left(x_{p}, y_{p}\right)$ are the coordinates of the scatterer $P$ prior to deformation, $\left(x_{p \_ \text {new }}, y_{p \_ \text {new }}\right)$ its coordinates after compression, and $\varepsilon$ the axial strain, positive for a dilatation and negative for a compression.

To increase the accuracy of the estimation, the simulated RF images were interpolated by a factor of 2 in both directions. Axial strain and lateral displacement fields were all computed using the following parameters. The region of interest $R_{1}$ measured 10 axial wavelengths by six interpolated lateral signals. The axial length came from previous experiments [22]

$$
\begin{aligned}
& {[\hat{t}]=[\hat{\alpha}, \hat{u}]=\arg \min _{\alpha, u}\left[f(\alpha, u)+\mu\left[\left(\frac{\alpha-\alpha_{\text {average }}}{\alpha_{\max }-\alpha_{\min }}\right)^{2}+\left(\frac{u-u_{\text {average }}}{u_{\max }-u_{\min }}\right)^{2}\right]\right]} \\
& \text { subject to }: A\left[\begin{array}{l}
\alpha \\
u
\end{array}\right] \leq b
\end{aligned}
$$

with

$$
t_{\text {average }}=\left[\begin{array}{l}
\alpha_{\text {average }}=\frac{1}{n} \sum_{j=1}^{n} R_{j} \hat{\alpha}_{j} \\
u_{\text {average }}=\frac{1}{n} \sum_{j=1}^{n} R_{j} \hat{u}_{j}
\end{array} \text { with }\left[\begin{array}{c}
\hat{\alpha}_{j} \\
\hat{u}_{j}
\end{array}\right]=\hat{t}_{j} \in V \cap \Omega\right.
$$




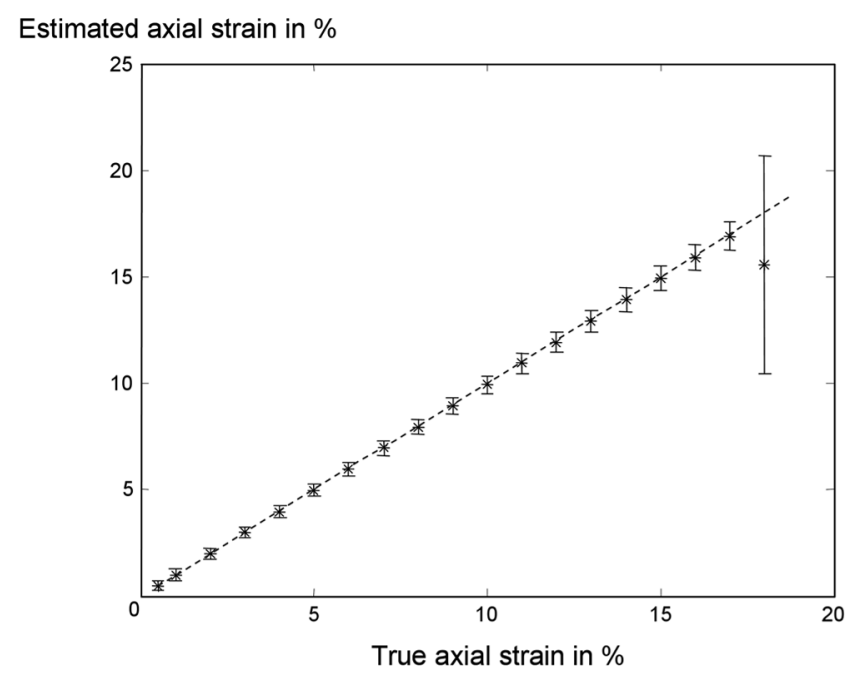

(a)

\section{Estimated axial displacement in $\mathrm{mm}$}

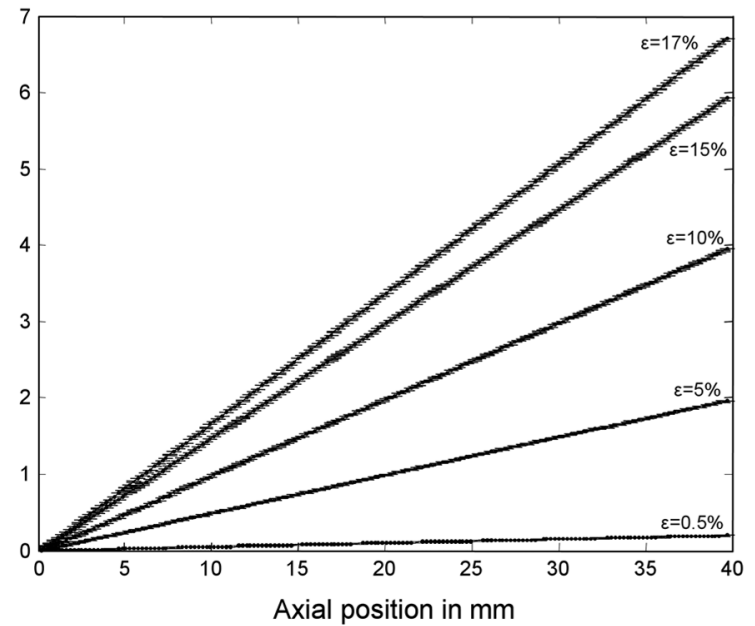

(c)

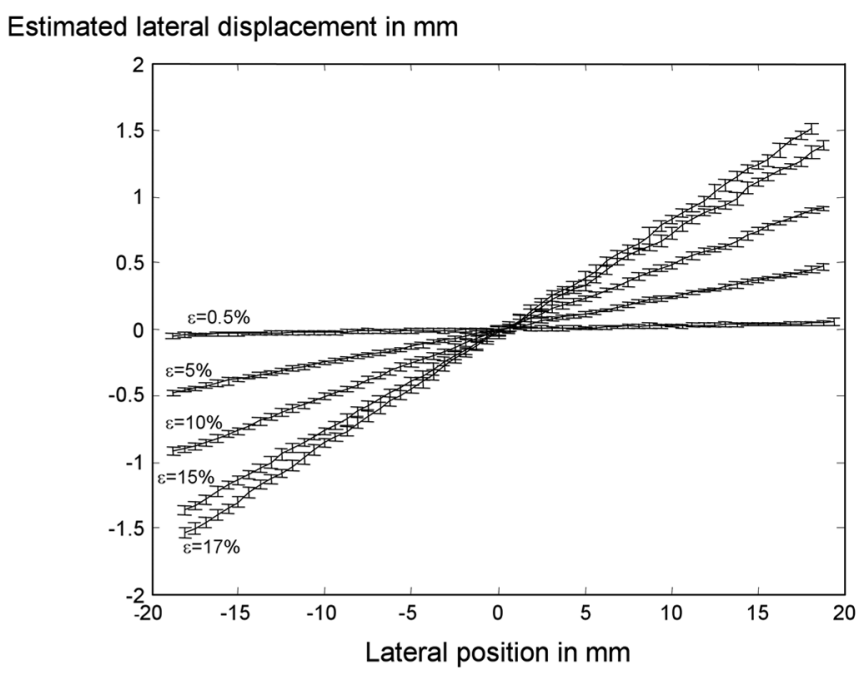

(b)

Mean normalized correlation coefficient

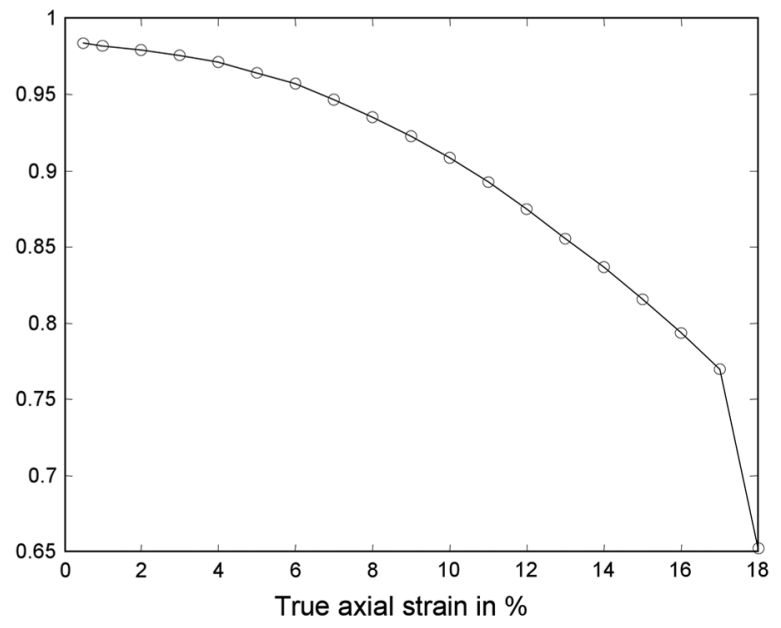

(d)

Fig. 3. Assessment of the method's performance with a mechanically homogeneous medium subjected to 19 compression levels, inducing $0.5 \%$ and from $1 \%$ to $18 \%$ (by $1 \%$ steps) of averaged axial strains. (a) Estimated mean axial strain and standard deviation as a function of the true strain. (b) Average lateral displacement profiles (along the lateral direction) and standard deviations for 0.5\%, 5\%,10\% 15\%, and 17\% strains applied. (c) Average axial displacement profiles and standard deviations for strains equal to $0.5 \%, 5 \%, 10 \%, 15 \%$, and $17 \%$. (d) Mean normalized correlation coefficients as a function of the compression level applied. The method computes accurate estimates for strains up to $17 \%$. Standard deviations along the lateral displacement profiles are higher than those along the axial displacement profiles but remain in good agreement with the theory.

that demonstrated it to be a good trade-off between estimation accuracy and spatial resolution. $R_{1}$ was displaced regularly in $I_{1}$ with an axial overlap of $75 \%$ and a lateral overlap of $60 \%$, while $R_{2}$ was adaptively displaced in $I_{2}$. Because of the range of axial strains considered, the bound values for the axial scaling factors were $\alpha_{\min }=0.8$ (maximal compression) and $\alpha_{\max }=1$ (no compression). Lateral displacement bounds were chosen such that a displacement of six interpolated signals on the left or on the right was allowed. This way the lateral displacement could be controlled while an erroneous estimation over an adjacent region could be compensated. Finally, the optimization was initialized to the center of the feasible domain $t_{0, n}^{(0)}=\left[\alpha_{0, n}^{(0)}=0.9 ; u_{0, n}^{(0)}=0\right]$ for regions at the probe-medium interface, while initialization for consecutive regions was performed as described in Section II-B2.
The threshold value for which the correction procedure detects an estimate as unreliable was set to $R_{\text {threshold }}=0.8$, generating $N b=10$ new minimization processes. In this section only, no local regularization was considered. Indeed, as discussed in Section II-B4, the regularization is dedicated to correct localized estimation errors, assuming that the axial strain and lateral displacement fields that result from steps in Sections II-B1, II-B2, and II-B3 are for the most part correctly estimated. This requires analyzing results at the end of the first three steps. Finally, it should be recalled that all estimated strain values were negative and for simplicity reasons, values will be displayed as positive, knowing that we measured a compression and not a dilatation.

Fig. 3(a) presents the mean and standard deviation of the estimated axial strains as a function of the true strains. We ob- 
serve that the method is able to accurately compute estimates for strains up to $17 \%$. The standard deviation increases slightly with the deformation but remains low over the range. Axial displacements were computed from axial strain estimates. Their mean profiles along the axial direction as well as standard deviations are reported in Fig. 3(c) for a few compression levels ( $\varepsilon=0.5 \%$, $5 \%, 10 \%, 15 \%$, and $17 \%$ ) for purposes of clarity. However, all compression levels were investigated and it was observed a very continuous evolution in axial displacement mean profiles from one level to another. Computed mean axial displacements are very close to the theoretical values. A slight increase in standard deviations with the compression level can be observed; however they remain on the order of $1 \mathrm{e}-3 \mathrm{~mm}$ for $\varepsilon=17 \%$. Mean profiles and standard deviations for lateral displacements are also provided [Fig. 3(b)]. As expected, their estimation is noisier than the estimation along the axial direction, but remains in good agreement with theoretical values. Finally, the mean correlation coefficient as a function of the compression level is displayed [Fig. 3(d)]. Its value, close to 1 for small strains, decreases with the applied compression to reach 0.8 for $16 \%$ strain. With increasing strains, signals are subjected to higher nonlinear amplitude and phase distortions that are not considered in our deformation model. Nevertheless, the technique proposed provides a good-quality estimation.

These results must, however, be cautiously interpreted, since simulations always remain ideal cases and the medium studied is mechanically homogeneous and therefore uniformly absorbs the compression. Biological tissues are unfortunately mainly heterogeneous, especially when they are pathological. Their compression will result in a wide range of strain variations. The load therefore needs to be carefully applied to avoid large areas of high strains $(>17 \%)$. Moreover, local strong medium heterogeneities may increase signal distortions, reducing the range of accurate strains with the use of medical data.

However, this range will remain sufficiently wide to investigate in vivo biological tissues. Previous studies concerning medical applications have demonstrated the potential of elastography, with elastograms exhibiting a narrower range of accurate strains [6], [9], [30].

The following results use local regularization when necessary. The parameters $R_{\text {reg }}$ and $\mu$ were empirically selected using phantom and biological tissue data. According to our observations, small areas of the displacement and strain fields that visually appear to suffer from an erroneous estimation were mainly characterized by a correlation coefficient that remained weak after the correction procedure. These considerations have led us to set $R_{\text {reg }}$ to 0.8 .

Determining $\mu$ was achieved by varying its value in the range $[0.25-100]$ and by investigating the regularization effects on the displacement and strain fields. We visually observed that for $\mu<8$, the regularization was too weak to smooth the areas concerned. For $\mu>40$, the parameter vector estimation was exclusively dominated by the regularization process. Finally, for $8 \leq \mu \leq 40$, a very slow continuous area smoothing was observed with increasing values for the regularization factor. Consequently $\mu$ was set to 30 .

3) Heterogeneous Mechanical Media Results: The ability of the proposed technique to image heterogeneous strain fields was investigated with two simulated linear elastic, nearly incompressible mechanical bodies. The first case we numerically created was a homogeneous cube $(E=50 \mathrm{kPa})$ containing a cylindrical inclusion that was twice as hard as the surrounding material $(E=100 \mathrm{kPa})$. This medium was subjected to a uniaxial load of $2 \mathrm{kPa}$. The second medium was a three-layer body, whose middle layer of Young's modulus $(E=50 \mathrm{kPa})$ was six times softer than the top and bottom layers $(E=300 \mathrm{kPa})$. This body was subjected to a uniaxial load of $7 \mathrm{kPa}$. Its deformation covered a wider range of strains than the example used in the first case. Both bodies measured $40 \times 40 \times 40 \mathrm{~mm}^{3}$.

The resulting displacement and strain fields from the embedded inclusion medium and the three-layer medium are presented in Figs. 4 and 5, respectively. For both, estimated fields are close to the theoretical values. In Fig. 4(b), the axial strain field brings out the hard inclusion with sharp boundaries, whereas it is not detectable in the conventional $B$-scan US image [Fig. 4(h)]. Regions located above and below the hard inclusion exhibit higher strains because of stress concentrations, as demonstrated with the theoretical field [Fig. 4(a)]. The corresponding axial displacement field has been deduced [Fig. 4(d)] and is in perfect agreement with the theory [Fig. 4(c)]. In the lateral direction, the displacement field also corroborates the theoretical values, but is noisier than the axial displacement field, because of the poor lateral resolution of the imaging system. Finally, as the objective function is based on the normalized correlation coefficient between a 2-D initial region and its compensated deformed version, this similarity criterion has been mapped. It remains strong throughout the entire image, achieving its highest values for less strained regions. The weakest values are reached at the inclusion boundaries, where the assumption of a constant strain over the region of interest is not valid. However, the correlation coefficient remains high and has been estimated at 0.96 on average.

Similar observations can be made with the fields resulting from the three-layer medium. Estimated strain and displacement fields are close to FEM distributions. Correlation coefficients are in the range [0.58-0.996], with more than $99 \%$ of them higher than 0.8 , resulting in a mean value of 0.94 .

\section{B. Results on Experimental Data}

1) Strain Imaging of a Tissue-Mimicking Phantom: RF US images were acquired from a parallelepipedic PVA cryogel phantom, measuring $3 \times 5 \times 4 \mathrm{~cm}^{3}$ and containing a harder cylindrical inclusion [Fig. 6(a)]. Polyvinyl alcohol (PVA) cryogel is a material whose stiffness increases by operating successive freeze-thaw cycles, adapted for constructing tissue-mimicking phantoms [36]. The surrounding medium and the inclusion were subjected to 1 and 3 freeze-thaw cycles, respectively. During the experiment, the bottom surface of the phantom lay on a support, while its top surface was compressed downward by lowering the US probe. The other four vertical exterior phantom surfaces were free to slip. The transducer was subjected to a $0.9-\mathrm{mm}$ vertical displacement inducing a mean global strain of $3 \%$. RF images were acquired with a $7-\mathrm{MHz}$ central frequency probe and sampled with a frequency of 50 MHz. Each image was composed of 128 RF A-lines. RF data 


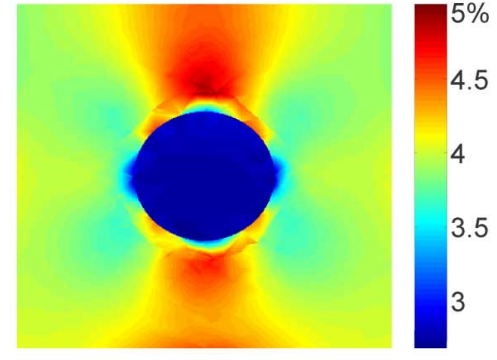

(a)

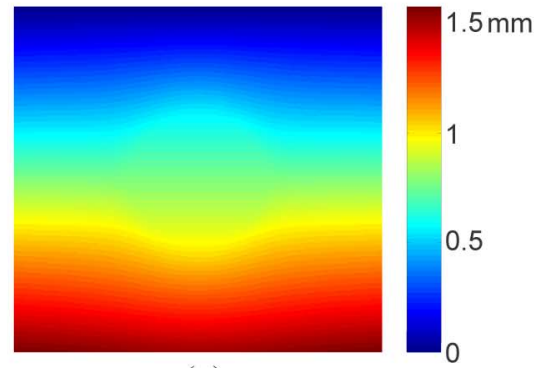

(c)

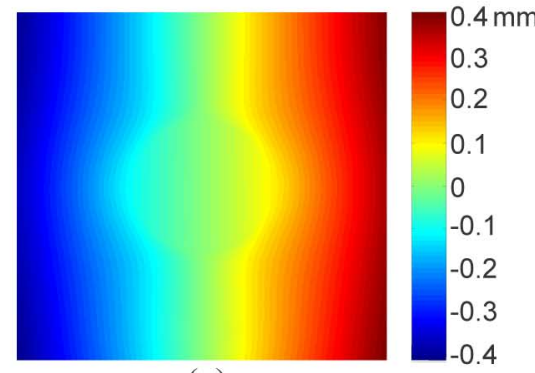

(e)

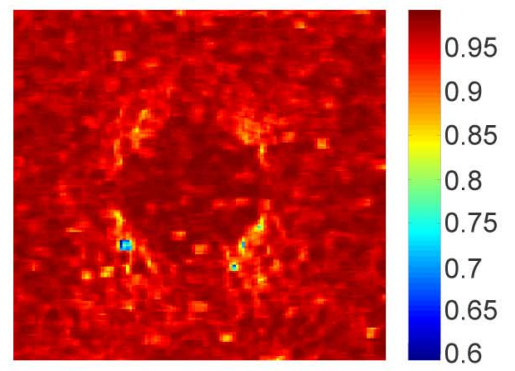

(g)

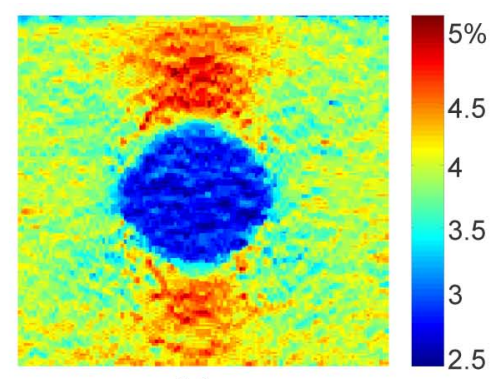

(b)

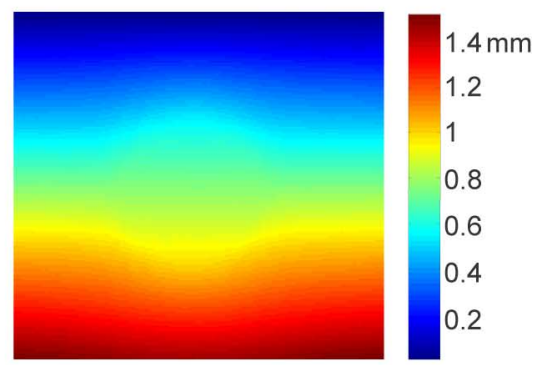

(d)

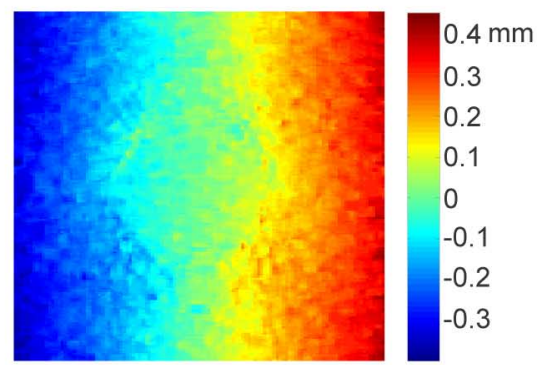

(f)

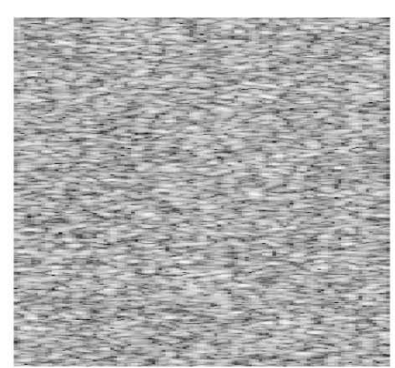

(h)

Fig. 4. Simulated inhomogeneous phantom with a harder inclusion. True: (a) axial strain, (c) axial displacement, (e) lateral displacement. Estimated: (b) axial strain, (d) axial displacement, (f) lateral displacement. (g) Normalized correlation coefficient map, (h) $B$-mode image.

were interpolated by a factor of 4 in the axial direction and by a factor of 2 in the lateral direction, prior to strain computation.

Results are presented in Fig. 6. While the inclusion is not revealed in the classical $B$-mode US image [Fig. 6(b)], it is clearly brought out with sharp boundaries in the axial elastogram [Fig. 6(c)]. The maximal axial displacement is estimated at $0.83 \mathrm{~mm}$, and the mean axial strain at $3.05 \%$, corroborating the experimental conditions. Lateral displacement estimation is noisy [Fig. 6(e)] but remains in agreement with the theoretical developments. Moreover, its significant amplitude demonstrates that it must be taken into account for a better estimation of the axial component. Indeed, we can observe that although noisy, considering the lateral displacement provides a correct estimation of the axial strain even at the lateral image extremities, where the lateral motion is the greatest. This level of quality could not be achieved with techniques that ignore this motion, such as 1-D methods. As illustrated with the elastogram provided in Fig. 6(g) and computed with a 1-D-scaling factor estimation technique [22], the axial strain computation is highly corrupted by signal decorrelation toward the image's lateral borders, resulting in erroneous values. This may lead to elastogram areas where a small lesion is undetectable because 


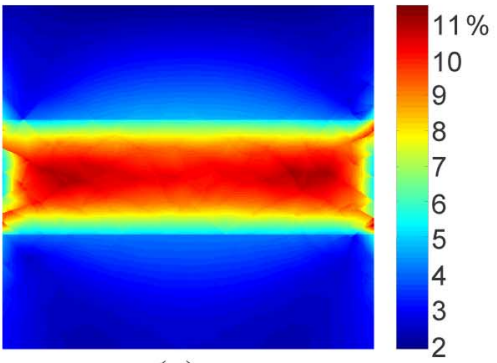

(a)

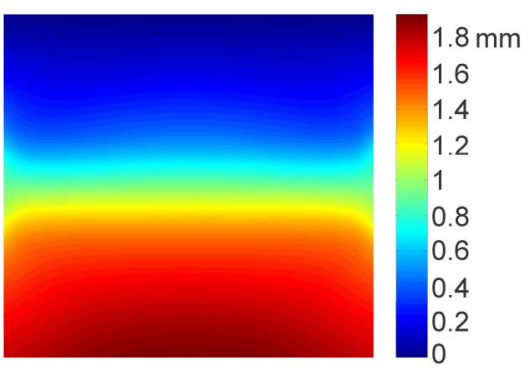

(c)

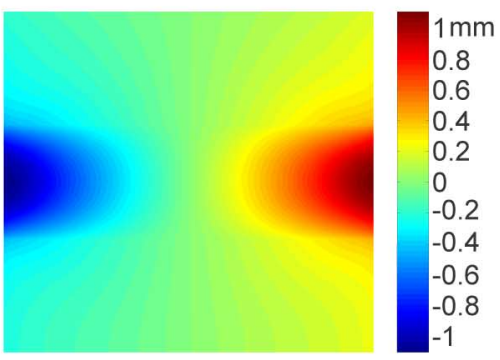

(e)

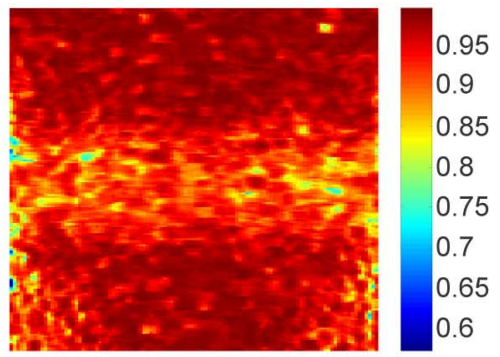

(g)

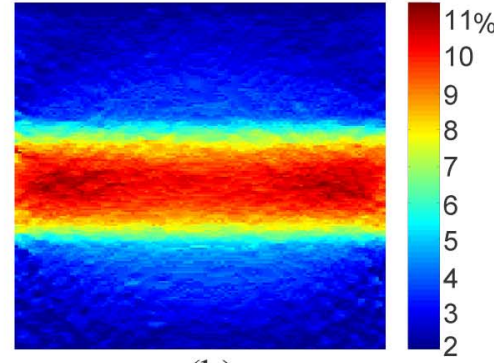

(b)

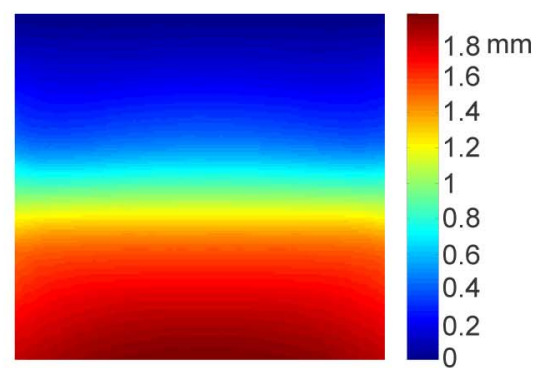

(d)

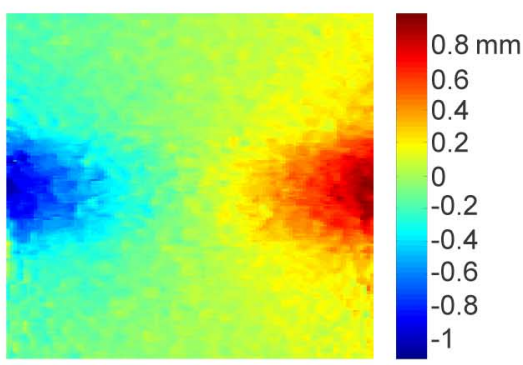

(f)

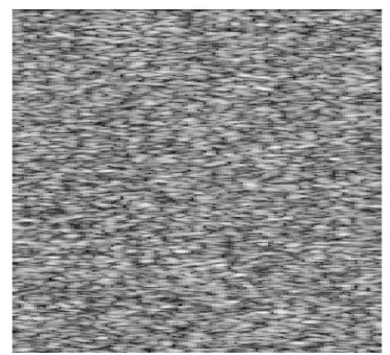

(h)

Fig. 5. Simulated three-layer phantom. True: (a) axial strain, (c) axial displacement, and (e) lateral displacement. Estimated: (b) axial strain, (d) axial displacement, and (f) lateral displacement. (g) Normalized correlation coefficient map. (h) $B$-mode image.

of the noise level. With such 1-D schemes, the loss of correlation induced by lateral motion cannot be compensated for by additional processing.

Finally, the distribution of the normalized correlation coefficient between an initial 2-D region and its deformed version, compensated for the lateral shift and axial scaling factor, is quasi-uniform and estimated on average at 0.89 [Fig. 6(f)].

2) Strain Imaging of In Vitro Bovine Livers: Our method was finally tested on experimental data from two cut specimens of bovine liver [Fig. 7(a)]. The first biological sample presented variable thicknesses with a maximum of $28 \mathrm{~mm}$. An embedded harder inclusion in agar gel measuring approximately $7.5 \mathrm{~mm}$ in diameter was created inside the soft tissue [Fig. 7(b)]. The same experimental protocol as described in the previous section was applied, with the two following differences. The vertical displacement of the probe to perform the compression was decreased to 0.2 and $0.4 \mathrm{~mm}$ because of the weak thickness of the tissue, emphasized by the significant precompression that needs to be applied to ensure sufficient contact between the transducer and the curved top surface of the specimen. Moreover, the sampling frequency was set at $200 \mathrm{MHz}$. Data were interpolated by a factor of 2 in the lateral direction.

Fig. 7 illustrates the axial strain fields for $0.2 \mathrm{~mm}$ [Fig. 7(d)] and $0.4 \mathrm{~mm}$ [Fig. 7(h)] of vertical displacements. The estimated 


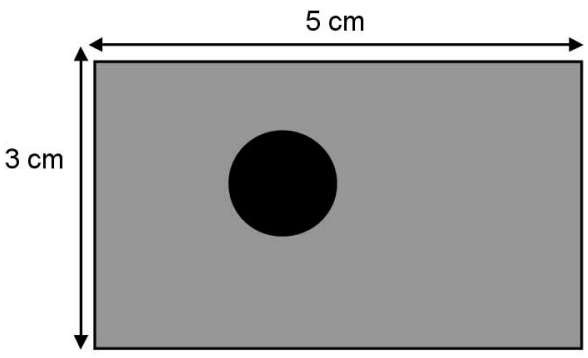

(a)

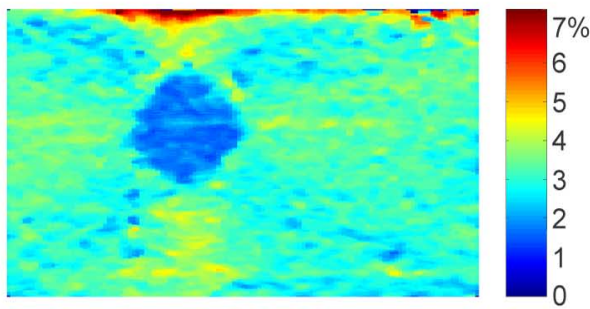

(c)

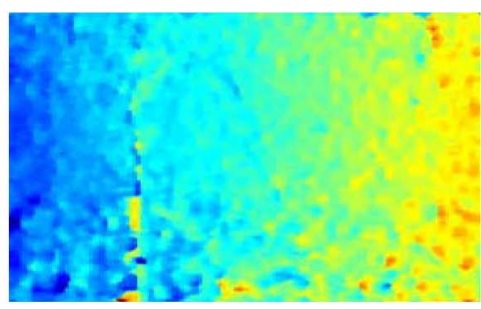

(e)

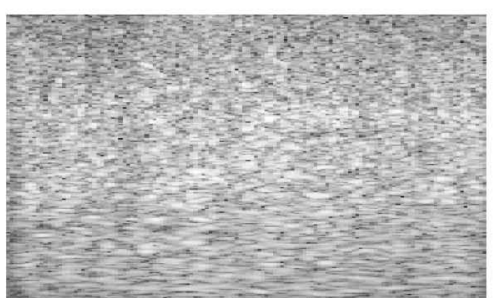

(b)

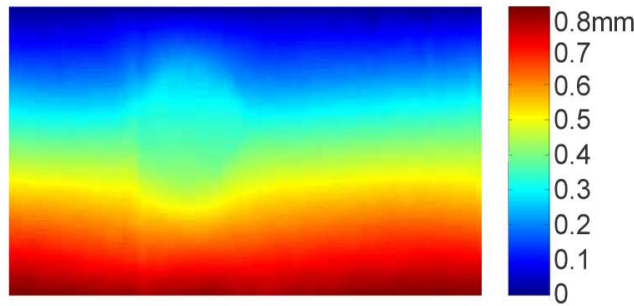

(d)

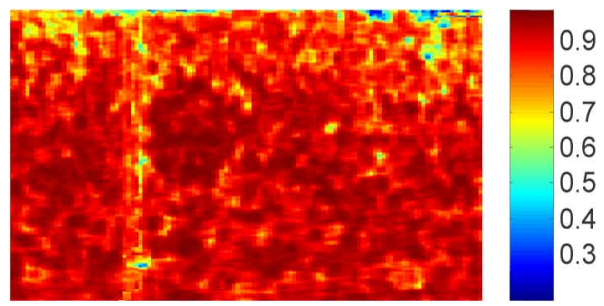

(f)

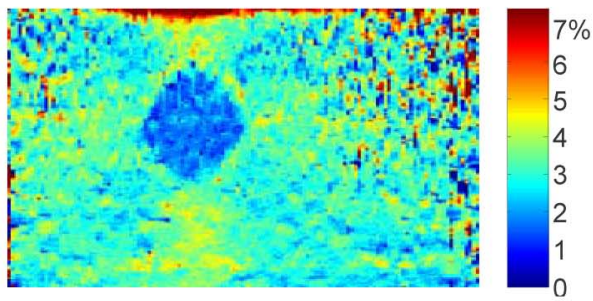

(g)

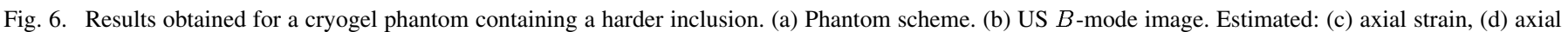

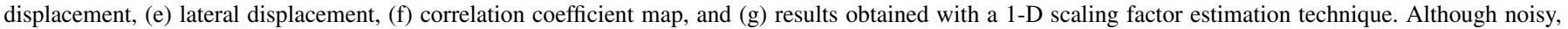

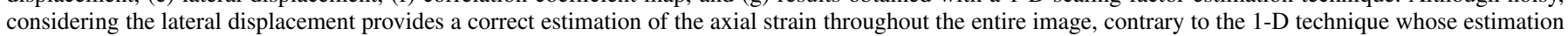
fails at the image extremities, where the lateral motion is the greatest.

axial [Fig. 7(e)] and lateral [Fig. 7(f)] displacement fields as well as the correlation coefficient map [Fig. 7(g)] are presented for the $0.2-\mathrm{mm}$ vertical displacement. However, similar results were obtained for the 0.4-mm motion. The results demonstrate that our technique can discriminate harder regions within soft biological tissues. Similar comments to those provided for the phantom can be made here. In particular, the inclusion is shown in the axial elastogram, with a clearly delimited shape. The estimated maximum axial displacements are in perfect agreement with the compression application conditions.

Finally, the normalized correlation coefficient field is quasiuniform with a mean of 0.97 and 0.915 for $0.2-\mathrm{mm}$ and $0.4-\mathrm{mm}$ vertical displacement, respectively, indicating the good similarity achieved between a 2-D region and its deformed compensated version.
The results from bovine liver sample \#2 containing a harder agar inclusion are presented in Fig. 8. This sample, whose maximum thickness reached $36 \mathrm{~mm}$ prior to precompression, was investigated with the same experimental conditions as sample \#1, except that the vertical displacement applied was set to $1 \mathrm{~mm}$.

We can observe that the lesion exhibits a much lower strain than the surrounding regions, making it clearly visible in the axial elastogram [Fig. 8(b)]. Moreover, the axial displacement field [Fig. 8(c)] deduced from the axial strain distribution has a maximum value that perfectly corroborates the conditions of the load application. The lateral displacement field [Fig. 8(d)] is greater than $0.6 \mathrm{~mm}$ at the lateral image extremities, demonstrating that it does indeed need to be considered. Finally, the mean normalized correlation coefficient has been estimated at 0.85 [Fig. 8(e)]. 


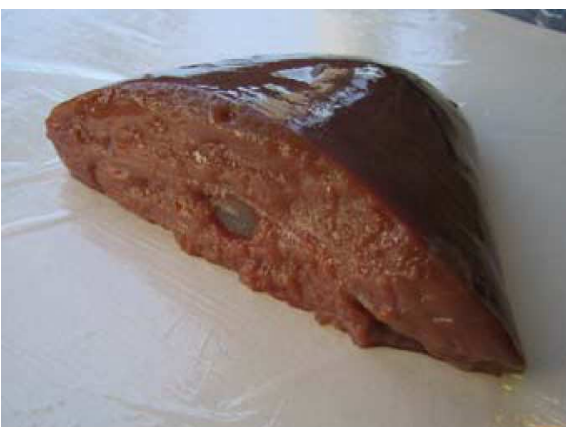

(a)

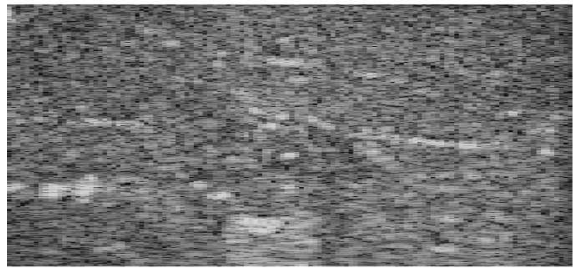

(c)

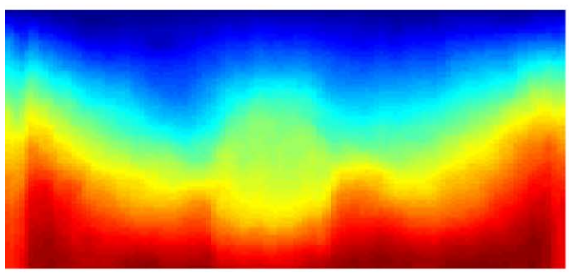

(e)

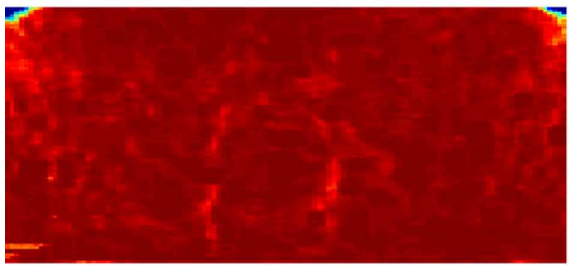

(g)

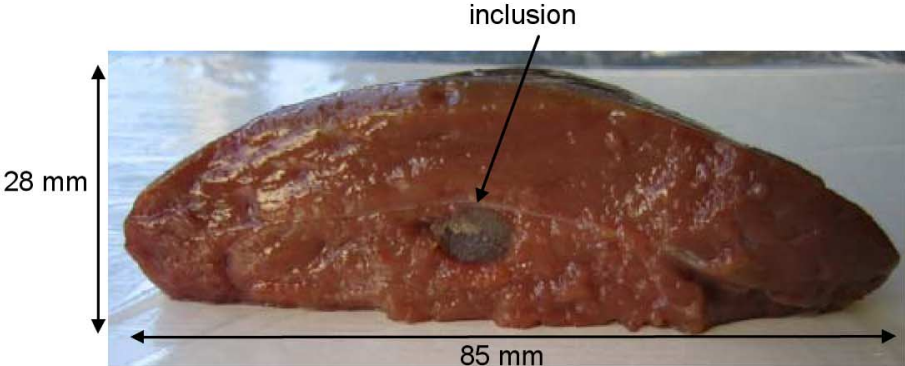

(b)

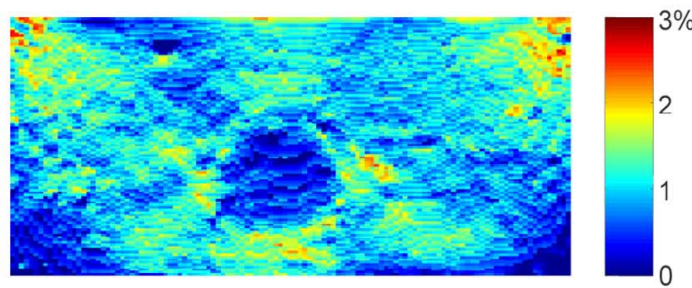

(d)
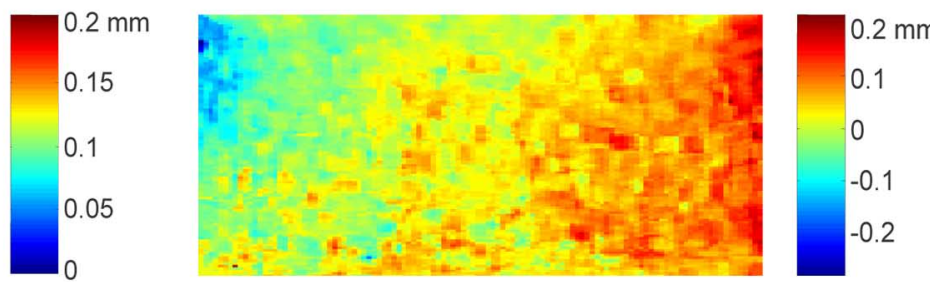

(f)

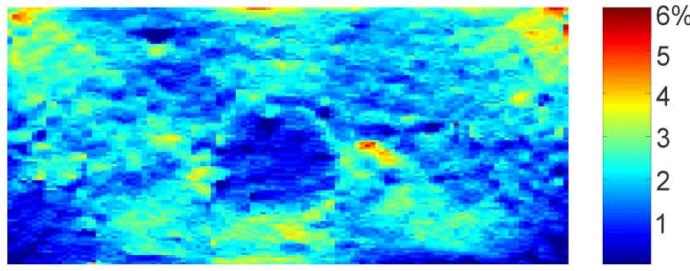

(h)

Fig. 7. Results from bovine liver sample \#1 within which is embedded a harder inclusion in agar. (a) Global view of the biological sample. (b) Photograph of the slice of interest. (c) US B-mode image. Estimated: (d) axial strain, (e) axial displacement, (f) lateral displacement, and (g) normalized correlation coefficient, for a vertical displacement of $0.2 \mathrm{~mm}$, and (h) axial strain for a $0.4-\mathrm{mm}$ probe motion applied. Whereas the agar inclusion is nearly undetectable in the $B$-mode image, it is clearly visible in the deformation fields. Moreover, the difference in range of axial strain fields (d) and (h) as well as the axial displacement distribution are in perfect agreement with the experimental conditions of the load application.

\section{CONCLUSION}

In this paper, a 2-D strain estimation algorithm was introduced, computing the axial strain while considering lateral motion. Contrary to most 2-D techniques that model the compression-induced local displacement as a 2-D shift, we also consider a scaling factor in the axial dimension. This leads to a method that is much more robust in terms of decorrelation noise and results in a larger range of accurate measurements.

To achieve maximum accuracy, the technique computes deformation parameters as those leading to the best possible match between the precompression and postcompression 2-D RF regions, when the latter are highly correlated. This is done through the constrained maximization of an objective function, defined as the normalized correlation coefficient between the initial 2-D $\mathrm{RF}$ acoustical region and the deformed region, compensated for the deformation parameters. When the correlation is lost, the estimation integrates an additional local smoothness constraint, imposing the continuity of resulting displacement and strain fields.

Two error sources for the model (1) should nevertheless be mentioned. The processed RF images inevitably contain an additive noise that can be modeled as a signal-independent, zero-mean, spatially uncorrelated process like electronic noise [18]. However, the similarity criterion used is known to be robust to such noise [37]. Moreover, with increasing strains, signals will be subjected to higher nonlinear amplitude and phase distortions that are not considered in our deformation model. This represents the major cause of decorrelation at larger strains.

The results on phantom and in vitro biological data demonstrate the ability of our technique to image deformation, providing information complementary to standard US images. 


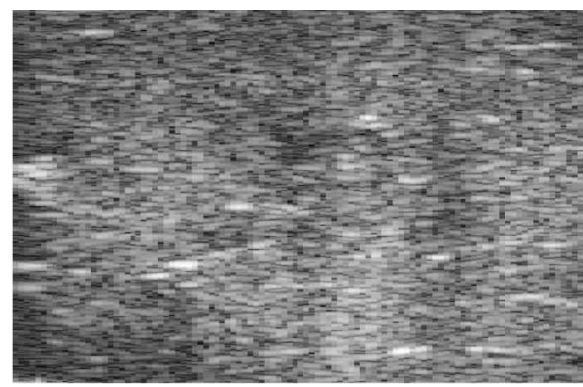

(a)

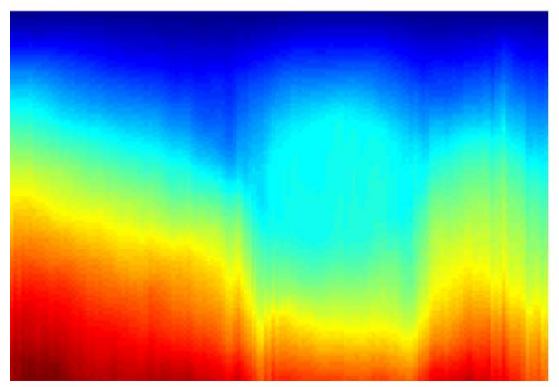

(c)

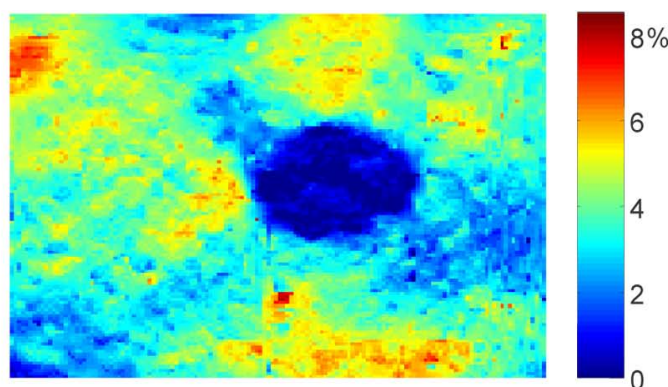

(b)
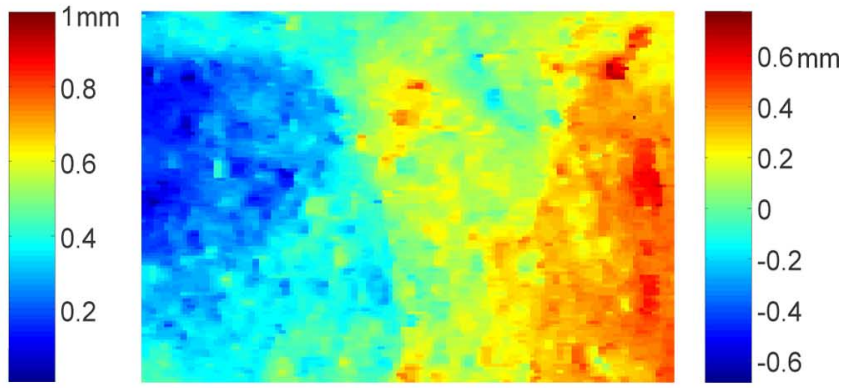

(d)

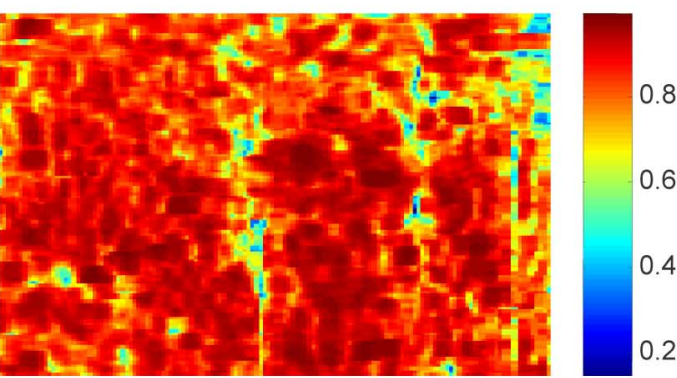

(e)

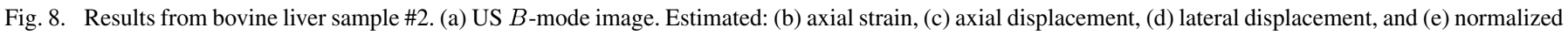
correlation coefficient for a 1-mm vertical displacement of the probe. Agar inclusion exhibits a much lower strain than the surrounding medium.

Compared to 1-D techniques, the algorithm described is characterized by a significant increase of computational costs. With the current implementation, the run time to compute one axial strain image and the corresponding lateral field is a few minutes on a PC (Pentium M 1.7-GHz Processor, 1GB. RAM). Developing a real-time method is beyond the scope of this paper. However, it has to be mentioned that the algorithm can be modified to support parallel computing. Investigating such implementation will be part of our future work.

In the present implementation, bounds of the feasible region and thresholds are constants. It should be possible to adapt them according to the estimated compression level. This could accelerate the convergence of the algorithm. Therefore these parameter values may evolve with future analysis. Finally, the present algorithm, based on the sequential quadratic programming methodology, allows the immediate addition of new constraints, whether linear or nonlinear. It can also be easily formalized in 3-D.

\section{APPENDIX}

A. The Sequential Quadratic Programming (sequential QP) Algorithm is a generalization of Newton's method, in that it finds a step away from the current point by minimizing a quadratic model of the problem.
Given initializations, $k=0, t_{0}, \lambda_{i, 0}, H_{0}=\left[\begin{array}{ll}1 & 0 \\ 0 & 1\end{array}\right]$ the technique consists in iteratively:

1) Forming and solving the (QP) subproblem to obtain the descent direction $d_{k}$ (see Appendix B).

2) Determining a step length $\rho_{k}$ to obtain a sufficient decrease in the objective function.

3) Set $t_{k+1}=t_{k}+\rho_{k} d_{k}$ and updates the Lagrange multipliers.

4) STOP if convergence (= satisfaction of the Kuhn-Tucker conditions).

5) Else compute $H_{k+1}$ (BFGS update), set $k:=k+1$ and go to 1$)$.

$B$. Form and Solve the $(Q P)$ Subproblem: The descent direction $d_{k}$ is computed as the solution of the associated QP subproblem. In our case, it corresponds to finding the constrained minimum of the quadratic approximation of the objective function since all constraints are linear

$$
\begin{aligned}
& \min _{d_{t}} \nabla f\left(t_{k}\right)^{T} \cdot d_{t}+\frac{1}{2} d_{t}^{T} \cdot H\left(t_{k}\right) \cdot d_{t} \\
& \text { subject to: } A\left(d_{t}+t_{k}\right) \leq b
\end{aligned}
$$

where $d_{t}=t-t_{k}$. 


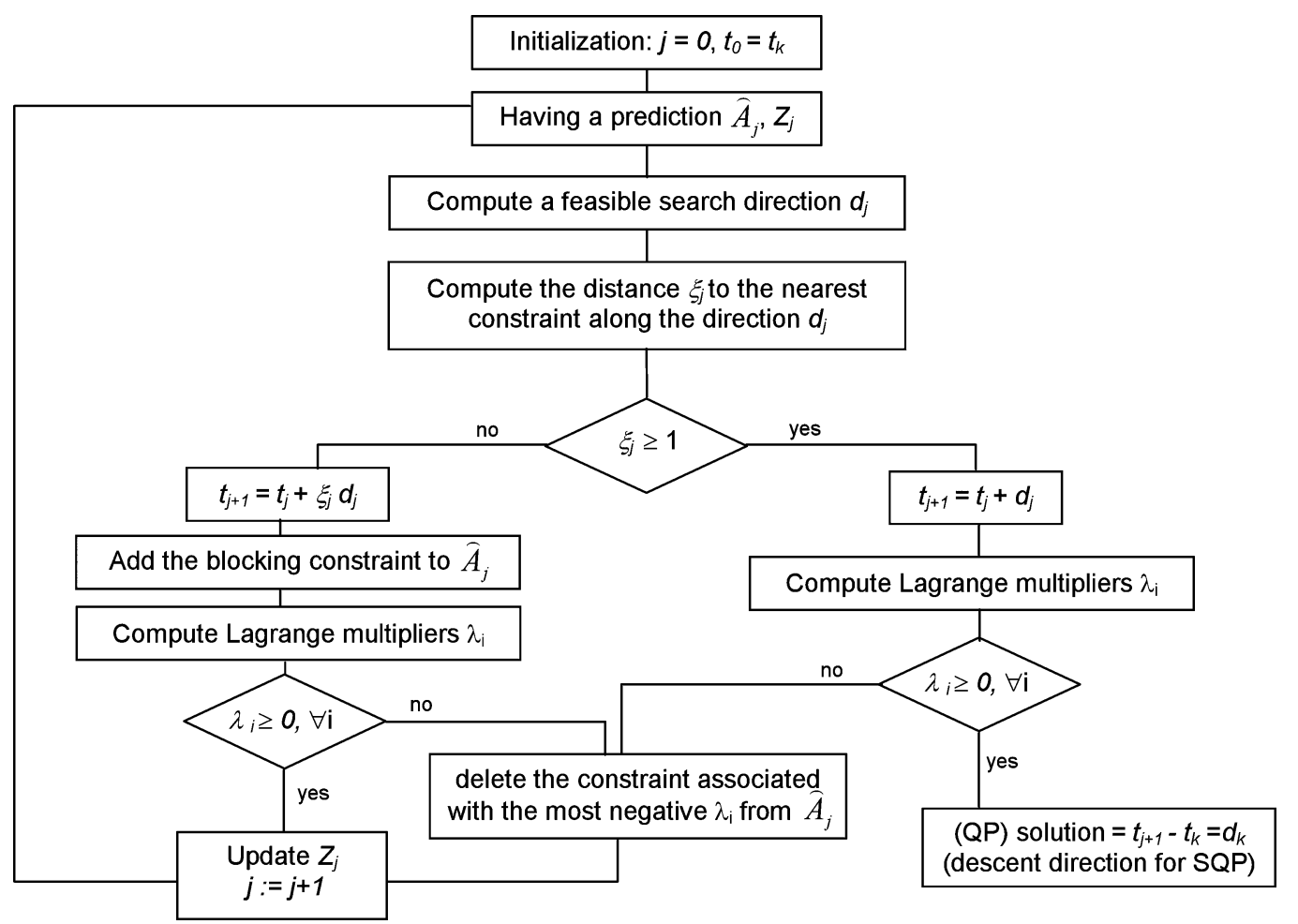

Fig. 9. QP solution computation.

This problem is solved by using an active set strategy. Active set methods are procedures that aim at identifying the constraints that will become active at the solution. Since it is not possible to know a priori which constraints will be active at the solution, these techniques are based on developing a prediction of the correct active set. And because the prediction could be wrong, the technique must also include procedures to modify it.

In the article, we denoted $\hat{A}$ the submatrix of $A$ containing the coefficients of the constraints active at the solution and $Z$ the matrix whose columns form a basis for the set of vectors orthogonal to the rows of $\hat{A}$. $Z$ thus defined the subspace of feasible search directions.

Similarly, we will denote $\hat{A}_{j}$ the prediction of the active set at the $j$ th iteration and $Z_{j}$ the corresponding subspace of feasible directions.

The technique works as shown in Fig. 9. In most cases, this process is completed in 1 or 2 iterations.

\section{REFERENCES}

[1] J. Ophir, I. Céspedes, H. Ponnekanti, Y. Yazdi, and X. Li, "Elastography: A quantitative method for imaging the elasticity of biological tissues," Ultrason. Imag., vol. 13, pp. 111-134, 1991.

[2] W. A. D. Anderson and J. M. Kissane, Pathology, 9th ed. St. Louis, MO: Mosby, 1953.

[3] T. A. Krouskop, T. M. Wheeler, F. Kallel, B. S. Garra, and T. Hall, "Elastic moduli of breast and prostate tissues under compression," $\mathrm{Ul}$ trason. Imag., vol. 20, pp. 260-274, 1998.

[4] B. S. Garra, E. I. Céspedes, J. Ophir, S. R. Spratt, R. A. Zuurbier, C. M. Magnant, and M. F. Pennanen, "Elastography of breast lesions: Initial clinical results," Radiology, vol. 202, pp. 79-86, 1997.

[5] L. Sandrin, B. Fourquet, J. M. Hasquenoph, S. Yon, C. Fournier, F. Mal, C. Christidis, M. Ziol, B. Poulet, F. Kazemi, M. Beaugrand, and R. Palau, "Transient elastography: A new noninvasive method for assessment of hepatic fibrosis," Ultrasound Med. Biol., vol. 29, no. 12, pp. $1705-1713,2003$
[6] C. L. De Korte, S. G. Carlier, F. Mastik, M. M. Doyley, A. F. W. Van Der Steen, P. Serruys, and N. Bom, "Morphological and mechanical information of coronary arteries obtained with intravascular elastography-Feasibility study in vivo," Eur. Heart J., no. 23, pp. 405-413, 2002.

[7] C. L. D. Korte, G. Pasterkamp, A. F. W. van der Steen, H. A. Woutman, and N. Bom, "Characterization of plaque components with intravascular ultrasound elastography in human femoral and coronary arteries in vitro," Circulation, vol. 102, pp. 617-23, 2000.

[8] E. Brusseau, J. Fromageau, G. Finet, P. Delachartre, and D. Vray, "Axial strain imaging of intravascular data: Results on polyvinyl alcohol cryogel phantoms and a carotid artery," Ultrasound Med. Biol., vol. 27, no. 12, pp. 1631-1642, 2001.

[9] R. Souchon, O. Rouviere, A. Gelet, V. Detti, S. Srinivasan, J. Ophir, and J. Y. Chapelon, "Visualisation of HIFU lesions using elastography of the human prostate in vivo: Preliminary results," Ultrasound Med. Biol., vol. 29, no. 7, pp. 1007-15, 2003.

[10] Y. Mofid, F. Ossant, F. Kathyr, M. Limberis, and F. Patat, "In vivo human skin elastography: Preliminary study," in Proc. World Congress Ultrasonics, 2003, pp. 205-208.

[11] J. L. Genisson, T. Baldeweck, M. Tanter, S. Catheline, M. Fink, L. Sandrin, C. Cornillon, and B. Querleux, "Assessment of elastic parameters of human skin using dynamic elastography," IEEE Trans. Ultrason., Ferroelect., Freq. Contr., vol. 51, no. 8, pp. 980-989, Aug. 2004.

[12] S. Emelianov, M. A. Lubinski, W. F. Weitze, R. C. Wiggins, A. R. Skovoroda, and M. O'Donnell, "Elasticity imaging for early detection of renal pathology," Ultrasound Med. Biol., vol. 21, no. 7, pp. 871-883, 1995.

[13] C. L. de Korte, A. F. W. van der Steen, B. H. J. Dijkman, and CT. Lancée, "Performance of time delay estimation methods for small time shifts in ultrasonic signals," Ultrasonics, vol. 35, pp. 263-274, 1997.

[14] X. Lai and H. Torp, "Interpolation methods for time-delay estimation using cross-correlation method for blood velocity measurement," IEEE Trans. Ultrason., Ferroelectr., Freq. Contr., vol. 46, no. 2, pp. 277-290, Mar. 1999.

[15] X. L. Xu, A. H. Tewfik, and J. F. Greenleaf, "Time delay estimation using wavelet transform for pulsed-wave ultrasound," Ann. Biomed. Eng., vol. 23, no. 5, pp. 612-621, 1995. 
[16] J. Luo, J. Bai, P. He, and K. Ying, "Axial strain calculation using a low-pass digital differentiator in ultrasound elastography," IEEE Trans. Ultrason., Ferroelect., Freq. Contr., vol. 51, no. 9, pp. 1119-1127, Sep. 2004.

[17] A. Pesavento, C. Perrey, M. Krueger, and H. Ermert, "A time-efficient and accurate strain estimation concept for ultrasonic elastography using iterative phase zero estimation," IEEE Trans. Ultrason., Ferroelect., Freq. Contr., vol. 46, no. 5, pp. 1057-1067, Sep. 1999.

[18] M. Bilgen and M. F. Insana, "Deformation models and correlation analysis in elastography," J. Acoust. Soc. Am., vol. 99, no. 5, pp. 3212-3224, 1996.

[19] M. Bilgen and M. F. Insana, "Error analysis in acoustic elastography. I. Displacement estimation," J. Acoust. Soc. Am., vol. 101, no. 2, pp. 1139-1146, 1997.

[20] S. K. Alam, J. Ophir, and E. E. Konofagou, "An adaptive strain estimator for elastography," IEEE Trans. Ultrason., Ferroelect., Freq. Contr., vol. 45, no. 2, pp. 461-472, Mar. 1998.

[21] M. Bilgen, "Wavelet-based strain estimator for elastography," IEEE Trans. Ultrason., Ferroelect., Freq. Contr., vol. 46, pp. 1407-1415, 1999.

[22] E. Brusseau, C. Perrey, P. Delachartre, M. Vogt, D. Vray, and H. Ermert, "Axial strain imaging using a local estimation of the scaling factor from RF ultrasound signals," Ultrason. Imag., vol. 22, no. 2, pp. 95-107, 2000.

[23] M. O'Donnell, A. R. Skovoroda, B. M. Shapo, and S. Y. Emelianov, "Internal displacement and strain imaging using ultrasonic speckle tracking," IEEE Trans. Ultrason., Ferroelect., Freq. Contr., vol. 41, pp. 314-324, 1994.

[24] X. Chen, M. J. Zohdy, S. Y. Emelianov, and M. O'Donnell, "Lateral speckle tracking using synthetic lateral phase," IEEE Trans. Ultrason., Ferroelect., Freq. Contr., vol. 51, no. 5, pp. 540-550, May 2004.

[25] E. E. Konofagou and J. Ophir, "A new elastographic method for estimation and imaging of lateral displacements, lateral strains, corrected axial strains and Poisson's ratio in tissues," Ultrasound Med. Biol., vol. 24, no. 8, pp. 1183-1199, 1998.
[26] P. Chaturvedi, M. F. Insana, and T. J. Hall, "2-D companding for noise reduction in strain imaging," IEEE Trans. Ultrason., Ferroelec., Freq. Contr., vol. 45, no. 1, pp. 179-191, Jan. 1998.

[27] P. Chaturvedi, M. F. Insana, and T. J. Hall, "Testing the limitations of 2-D companding for strain imaging using phantoms," IEEE Trans. Ultrason., Ferroelec., Freq. Contr., vol. 45, no. 4, pp. 1022-1031, Jul. 1998.

[28] R. L. Maurice, J. Ohayon, Y. Frétigny, M. Bertrand, G. Soulez, and G. Cloutier, "Non-invasive vascular elastography: Theoretical framework," IEEE Trans. Med. Imag., vol. 23, no. 2, pp. 164-180, Feb. 2004.

[29] R. L. Maurice and M. Bertrand, "Lagrangian speckle model and tissuemotion estimation-Theory," IEEE Trans. Med. Imag., vol. 18, no. 7, pp. 593-603, Jul. 1999.

[30] C. Pellot-Barakat, F. Frouin, M. F. Insana, and A. Herment, "Ultrasound elastography based on multiscale estimations of regularized displacement fields," IEEE Trans. Med. Imag., vol. 23, no. 2, pp. 153-63, Feb. 2004.

[31] P. T. Boggs and J. W. Tolle, "Sequential quadratic programming," Acta Numerica, vol. 4, pp. 1-51, 1996.

[32] P. E. Gill, W. Murray, and M. H. Wright, Practical Optimization. New York: Academic, 1981.

[33] F. Heitz, P. Perez, and P. Bouthemy, "Multiscale minimization of global energy functions in some visual recovery problems," CVGIP: Image Understand., vol. 59, no. 1, pp. 125-134, 1994.

[34] Y. C. Fung, Biomechanics-Mechanical Properties of Living Tissues, 2nd ed. New York: Springer-Verlag, 1993.

[35] H. Du, J. Liu, C. Pellot Barakat, and M. F. Insana, "Optimizing multicompression approaches to elasticity imaging," IEEE Trans. Ultrason., Ferroelect., Freq. Contr., vol. 53, no. 1, pp. 90-99, Jan. 2006.

[36] K. C. Chu and B. K. Rutt, "Polyvinyl alcohol cryogel: An ideal phantom material for MR studies of arterial flow and elasticity," Magn. Reson. Med., vol. 37, no. 2, pp. 314-319, 1997.

[37] F. Viola and W. F. Walker, "Comparison of time-delay estimators in medical ultrasound," in Proc. IEEE Ultrason. Symp., Oct. 2001, vol. 2, pp. $1485-1488$. 